\title{
Phase transition in anisotropic holographic superfluids with arbitrary dynamical critical exponent $z$ and hyperscaling violation factor $\alpha$
}

\author{
Miok Park ${ }^{1, a}$, Jiwon Park ${ }^{2, b}$, Jae-Hyuk Oh ${ }^{2, c}$ \\ ${ }^{1}$ Korea Institute for Advanced Study, Seoul 02455, Korea \\ ${ }^{2}$ Department of Physics, Hanyang University, Seoul 133-791, Korea
}

Received: 17 April 2017 / Accepted: 13 November 2017 / Published online: 28 November 2017

(C) The Author(s) 2017. This article is an open access publication

\begin{abstract}
Einstein-scalar- $U$ (2) gauge field theory is considered in a spacetime characterized by $\alpha$ and $z$, which are the hyperscaling violation factor and the dynamical critical exponent, respectively. We consider a dual fluid system of such a gravity theory characterized by temperature $T$ and chemical potential $\mu$. It turns out that there is a superfluid phase transition where a vector order parameter appears which breaks $S O$ (3) global rotation symmetry of the dual fluid system when the chemical potential becomes a certain critical value. To study this system for arbitrary $z$ and $\alpha$, we first apply Sturm-Liouville theory and estimate the upper bounds of the critical values of the chemical potential. We also employ a numerical method in the ranges of $1 \leq z \leq 4$ and $0 \leq \alpha \leq 4$ to check if the Sturm-Liouville method correctly estimates the critical values of the chemical potential. It turns out that the two methods are agreed within 10 percent error ranges. Finally, we compute free energy density of the dual fluid by using its gravity dual and check if the system shows phase transition at the critical values of the chemical potential $\mu_{\mathrm{c}}$ for the given parameter region of $\alpha$ and $z$. Interestingly, it is observed that the anisotropic phase is more favored than the isotropic phase for relatively small values of $z$ and $\alpha$. However, for large values of $z$ and $\alpha$, the anisotropic phase is not favored.
\end{abstract}

\section{Contents}

1 Introduction . . . . . . . . . . . 1

2 Holographic model . . . . . . . . . . . . . . 4

3 Analytic and numerical approaches to the critical points 5

\footnotetext{
a e-mail: miokpark@kias.re.kr

be-mail: minerva1993@gmail.com

c e-mail: jack.jaehyuk.oh@gmail.com
}

4 Numerics . . . . . . . . . . . . . . . 6

4.1 Numerical solutions of $\omega(\xi)$ and $b(\xi) \quad \ldots .6$

4.2 Free energy density . . . . . . . . . . . . 7

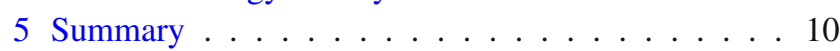

References . . . . . . . . . . . . . . . . 11

\section{Introduction}

Black hole thermodynamics implies that the black hole entropy is proportional to its horizon area. Entropy is an extensive quantity and so is comparable to the system volume. In fact, it is conjectured that there is a one to one mapping between the physical degrees of freedom or entropy inside the black hole being proportional to the black hole volume and those on its horizon which is a codimension one spacetime. This conjecture is called the holographic principle.

One of the examples of the holographic principle is the AdS/CFT correspondence. The AdS/CFT correspondence is a precise map between two different theories. One is a gravity theory defined in asymptotically $\operatorname{AdS}_{d+1}$. The other is its dual conformal field theory (CFT) defined on the AdS boundary, which is a $d$-dimensional spacetime without any gravitational fields. The pure AdS space corresponds to the vacua of the dual CFT. There are two different kinds of excitations on the gravity side. One kind is a normalizable mode of excitations, which corresponds to a non-trivial state in the dual CFT, and the other kind is a non-normalizable mode of excitations, which is coincident with a deformation with a certain operator in the dual CFT.

The AdS/CFT correspondence has lead to various applications on strongly coupled field theories. Especially, it is applied to explore (conformal) fluid dynamics (fluid/gravity duality) and condensed matter theories (AdS/CMT) $[1,8]$. 
The precise map of fluid/gravity duality is given in [20]. The claim in [20] is that there is a one to one correspondence between the following two theories. One is a conformal fluid dynamics with excitations of the temperature $T(\omega, \vec{k})$, the 4-velocity $u_{\mu}(\omega, \vec{k})$ and the charge density $\rho(\omega, \vec{k})$ where the frequency and the momenta of such a theory are much smaller than the inverse of the mean free path $\left(l_{\mathrm{mfp}}\right)$, namely, $(\omega, \vec{k}) \ll l_{\mathrm{mfp}}^{-1}$. The other is a dual gravity theory whose classical geometry is a locally boosted black brane solution slowly varying along the boundary directions, namely, its wave length $\lambda \gg r_{0}$, where the $r_{0}$ is the black brane horizon. Due to this property, it is possible that one gets the locally boosted black brane solution with a power expansion of small derivatives along the boundary directions. The geometry is characterized by the Hawking temperature of the black brane $T\left(x^{\mu}\right)$, 4-velocity $u_{\mu}\left(x^{\mu}\right)$ and (R)-charge density $\rho\left(x^{\mu}\right)$. The Einstein equations in the dual gravity provide constraints on these quantities as $\nabla_{\mu} T^{\mu \nu}=0$ and $\nabla_{\mu} J^{\mu}=0$, where $T^{\mu \nu}=T^{d}\left(x^{\alpha}\right)\left(g^{\mu \nu}\left(x^{\alpha}\right)+4 u^{\mu}\left(x^{\alpha}\right) u^{\nu}\left(x^{\alpha}\right)\right)+O\left(r_{0} \partial_{\mu}\right)$ and $J_{\mu}=\rho\left(x^{\alpha}\right) u_{\mu}\left(x^{\alpha}\right)+O\left(r_{0} \partial_{\mu}\right)$, which are called the boundary energy-momentum tensor and charge current, respectively.

An interesting research topic along these directions is the thermodynamic phase transition from normal fluids/conductor to superfluids/conductor. This holographic superfluid/superconductor phase transition is accompanied by a condensation (appearance of an order parameter) such as the appearance of Cooper pairs in the superconducting phase. The condensation breaks a global symmetry of the system. Therefore, in the development of AdS/CMT and fluid/gravity duality, the observation of several kinds of symmetry breaking mechanisms in a gravitational system plays an important role. The first idea that a black hole describes holographic superconductor/superfluids is suggested by the observation that the RN-AdS black hole is possibly unstable under a complex scalar perturbation below a certain Hawking temperature. Below that temperature the gravitational system presents its scalar hair outside of the black hole horizon in [3]. Based on this mechanism, the holographic superconductor model was established in [2], which shows a complex scalar field condensation resulting from a spontaneous symmetry breaking of global U(1) and it corresponds to an order parameter in the second order phase transition via a holographic interpretation.

Another type of holographic superconductor/superfluidity models is also investigated in the asymptotically charged$\mathrm{AdS}_{4}$ spacetime coupled to $\mathrm{SU}(2)$ non-Abelian gauge field in [4]. This type of the holographic models is proposed to explore $p$-wave superconductor/superfluidity, where their order parameters are vector-like. Some properties to the holographic dual description, such as the speed of second sound or the conductivity, are studied in asymptotically $\mathrm{AdS}_{5}$, by taking the probe limit in [18]. This model assumes that a chemical potential is given in the third isospin direction and accordingly it has a charge density as a response. Interestingly, below a certain temperature $T_{c}$, an additional current starts to be induced in a spatial direction, denoted $\left\langle j_{1}^{x_{1}}\right\rangle$. This current breaks $\mathrm{SU}(2)$ gauge symmetry and also the rotational symmetry $(\mathrm{SO}(3))$ of the system to $\mathrm{U}(1)$.

In the dual field theory it plays the role of the order parameter for the second order phase transition. The holographic dual of the anisotropic fluid dynamics is described by excitations in the background of an asymptotically RN-AdS black brane solution obtained from Einstein-SU(2) YangMills theory defined in 5-dimensional space. In the spatially isotropic phase, the temporal part of the Yang-Mills fields, $A_{t}^{3}$, is non-zero only, but in the anisotropic phase, a spatial part of the Yang-Mills fields arises, $A_{x_{1}}^{1}$, together with the temporal part. ${ }^{1}$ In $[8,18]$, it is found that the phase transition occurs at the chemical potential ${ }^{2} \mu_{\mathrm{c}}=4$ and the $\left\langle j_{1}^{x_{1}}\right\rangle$ starts to appear in the dual fluid system. Near the critical point, it is assumed that the current $\left\langle j_{1}^{x_{1}}\right\rangle$ takes a small value suppressed by a dimensionless small parameter $\epsilon$, the free energy can be analytically computed from the dual gravity with the power expansion of the parameter $\epsilon$. It is proved that the anisotropic phase is thermodynamically favorable when $\mu \geq 4$.

Beyond considering the asymptotically AdS spacetime, the applications of the holography to strongly correlated systems have inspired one to consider various gravitational systems such as the Lifshitz spacetime, hyperscaling violation geometry and so on. The Lifshitz spacetime was firstly introduced to realize temporal anisotropy emerging from quantum critical phenomena associated with continuous phase transitions in [5]. In the vicinity of the critical point, time scales differently from space,

$t \rightarrow \lambda^{z} t, \quad \vec{x} \rightarrow \lambda \vec{x}$,

where $z$ is called the dynamical critical exponent, and its dual geometrical realization can be written as

$\mathrm{d} s^{2}=-r^{2 z} \mathrm{~d} t^{2}+\frac{\mathrm{d} r^{2}}{r^{2}}+r^{2} \mathrm{~d} \vec{x}^{2}$,

where one restores the conformal invariance by taking $z=1$.

Furthermore, the gravitational action having the Abelian gauge fields coupled to the dilaton field is allowed to make a more general extension for the Lifshitz spacetime to have an overall hyperscaling factor $\alpha$ and so as not to be invariant under scaling,

\footnotetext{
${ }^{1}$ In $A_{x_{1}}^{1}$, the superscript indicates the adjoint index of SU(2) gauge group and $x_{1}$ is one of the spatial directions along the AdS boundary

2 This chemical potential, $\mu$, is dimensionless, which is seen by a rescaling transformation of the dimensionful chemical potential, $\tilde{\mu}$, with the black brane horizon $r_{0}$. The precise formula is given by (22).
} 
$\mathrm{d} s^{2} \rightarrow \lambda^{-\alpha} \mathrm{d} s^{2}$,

and the extended metric takes the form

$\mathrm{d} s^{2}=r^{2 \alpha}\left(-r^{2 z} \mathrm{~d} t^{2}+\frac{\mathrm{d} r^{2}}{r^{2}}+r^{2} \mathrm{~d} \vec{x}^{2}\right)$.

Some properties of this spacetime were studied in $[13,14,21-$ 28].

In previous research, the thermodynamic phase transition from the spatially isotropic to anisotropic states has widely been studied in the background of the charged-AdS black brane spacetime, where $z=1$ and $\alpha=0$. In this note, we consider more general spacetimes, showing arbitrary $z$ and $\alpha$, and assume that the system is near the critical point, $\mu=\mu_{\mathrm{c}}$. Near the critical chemical potential, the current $\left\langle j_{1}^{x_{1}}\right\rangle$ starts to be induced. To deal with this current, we assume that the amplitude of the current is small and so is suppressed by a dimensionless small parameter, $\epsilon$. We solve this system order by order in the power expansion of the parameter $\epsilon$. Our purpose is to find the numerical/analytic values of the $\mu_{\mathrm{c}}$ for generic $z$ and $\alpha$ and to compute the free energy density to check the thermodynamically favored state. To construct such a holographic model, we consider Einstein-scalar- $U(2)^{3}$ theory, which gives the background geometry presenting asymptotically hyperscaling violation and Lifshitz scale symmetry. For technical reasons, we take the limit that the Yang-Mills coupling constant, $g_{Y M}$, is large and the 5-dimensional gravity constant, $\kappa_{5}$, is small but their ratio, $\frac{\kappa_{5}}{g_{Y M}} \rightarrow 0$. This limit ensures that there is no back reaction from the Yang-Mills fields to the other ones. Namely, we consider a "probe limit", where we solve the Yang-Mills equations only, which become non-trivial near the critical points.

To analyze this system, we apply two different methods: analytic and numerical methods. First of all, we discuss the analytic method.

It may be very difficult or almost impossible to get analytic solutions of the Yang-Mills field equations near the critical point. Therefore, we focus on getting the critical value of the chemical potential. The analytic approach that we use is by Sturm-Liouville theory. The structure of the Yang-Mills equations with a certain boundary condition, $B(\psi(x))=0$ (where $x=0$ or $x=1$ ) has the following form:

$\mathcal{O}(x) \psi_{i}(x, \lambda)=\lambda_{i} \psi_{i}(x, \lambda)$

\footnotetext{
${ }^{3} U(2)$ means that $U(1) \times S U(2)$. In the dual gravity theory, we have two different gauge fields, $U(1)$ and $S U(2)$ gauge fields. We combine these and address those $U(2)$. It could be somewhat misapprehensive, since the spatial anisotropic phase is induced by a normalizable mode of $S U(2)$ gauge fields only, whereas the $U(1)$ gauge fields are just a background in our probe limit.
}

where the $\mathcal{O}(x)$ is a differential operator, the $\lambda_{i}$ are the eigenvalues of the operator (which corresponds to the $\mu_{\mathrm{c}}$ ) and $\psi_{i}(x, \lambda)$ is the eigenfunction (which corresponds to the Yang-Mills field $A_{x_{1}}^{1}$ ), where $i=0,1, \ldots, n$ with a certain $n \in \mathcal{Z}_{+}$. The Sturm-Liouville theory is meant to solve such differential equations with the undetermined parameters, $\lambda_{i}$ in the equations. The solutions exist only when the $\lambda_{i}$ attain appropriate values.

A difficulty in dealing with the Yang-Mills equations is that it is very unlikely to get analytic solutions of those. However, one can estimate the lowest eigenvalue of the system, even if one does not know the exact forms of the eigenfunctions $\psi_{i}$, by employing the following fact. Suppose that the eigenvalues are real, then one can relabel the eigenvalues and eigenfunctions for them to satisfy the condition that $\lambda_{i}>\lambda_{j}$ if $i>j$. An arbitrary (normalized) function, $\psi$, satisfying the boundary condition, $B(\psi(x))=0$ at $x=0$ or $x=1$, can be expressed by a summation of the eigenfunctions by assuming completeness of the eigenfunctions. Namely, $\psi=\sum_{i=0}^{i=n} a_{i} \psi_{i}$ with certain complex numbers, ${ }^{4} a_{i}$ satisfying $\sum_{i=0}^{i=n}\left|a_{i}\right|^{2}=1$. Then the expectation value of the operator $\mathcal{O}(x)$ from the $\psi,\langle\mathcal{O}(x)\rangle_{\psi}$ is given by $\sum_{i=0}^{i=n} \lambda_{i}\left|a_{i}\right|^{2}$, which is always greater than $\lambda_{0}$. Namely, the quantity $\langle\mathcal{O}(x)\rangle_{\psi}$ provides an upper bound of the lowest eigenvalue $\lambda_{0}$. This is called a variational principle [15].

The last question in the process is to choose the $\psi$ in a smart way which provides a very close (but no cigar) value to the $\mu_{\mathrm{c}}$. We have chosen $A_{x_{1}}^{1}(\xi) \sim \xi^{3 \alpha+3 z-1} F(\xi)$, where the factor $\xi^{3 \alpha+3 z-1}$ ensures the asymptotic behavior near the hyperscaling violation and Lifshitz boundary as $\xi \rightarrow 0$, where $\xi$ is a new radial variable such that $\xi \sim \frac{1}{r}$. We assume that $F(\xi)$ is a polynomial in $\xi$ as $F(\xi)=\sum_{i=0}^{\infty} b_{i} \xi^{i}$. We tune the coefficients $b_{i}$ to get the minima of the upper bounds of the $\mu_{\mathrm{c}}$. Then those values would be the closest to the critical chemical potentials.

In order to numerically find a critical value of $\mu$, we solve the coupled Yang-Mills equations of the $A_{x_{1}}^{1}$ and $A_{t}^{3}$ with appropriate boundary conditions at the black hole horizon and the asymptotic region for a fixed value $z$ and $\alpha$ by using a shooting method. Then we compare the Sturm-Liouville results with the numerical one. This is one of our main results, which is given in Fig. 1. In Fig. 1a, we plot the critical values of the chemical potential with the solid lines (analytic approach) and dashed lines (numerics) for $\alpha=0,1,2,3,4$ from below in order. They present a monotonically increasing behavior as $z$ increases for fixed values of $\alpha$. In Fig. 1b, we plot the critical values of the chemical potential $\mu_{\mathrm{c}}$ as the $\alpha$ increases for values of $z=1,2,3,4,5$ from below in order.

\footnotetext{
${ }^{4}$ For our case, $a_{i}$ are real numbers since the Yang-Mills fields are real.
} 
We also compute the free energy from the Euclideanized dual gravity on-shell action to compare that of the isotropic state with anisotropic state by using the numerical solutions of Yang-Mills fields. Interestingly, the numerical results show that the anisotropic state is thermodynamically favored only in a certain region in the $\alpha$ versus $z$ plane. In another region that we explore, the free energy is severely oscillating and we cannot conclude which state is favored thermodynamically. The result is addressed and the details are discussed in Sect. 4.2

This note is organized as follows. In Sect. 2, we discuss our holographic setting of the gravity model which gives asymptotically hyperscaling violation, Lifshitz scaling symmetry and spatial anisotropy for the critical values of the chemical potential. In Sect. 3, we explain our analytic method and in Sect. 4, we demonstrate numerical methods and the results. In Sect. 5, we summarize our work.

\section{Holographic model}

We start with the bulk action

$$
\begin{aligned}
S= & \frac{1}{\kappa_{5}^{2}} \int \mathrm{d}^{5} x \sqrt{-g}\left[R-\frac{1}{2} g^{M N} \partial_{M} \phi \partial_{N} \phi\right. \\
& +\frac{V_{0}}{L^{2}} e^{\gamma \phi}-\frac{\kappa_{5}^{2}}{4 g_{U}^{2}} e^{\lambda_{U} \phi} F_{M N} F^{M N} \\
& \left.-\frac{\kappa_{5}^{2}}{4 g_{Y M}^{2}} e^{\lambda_{Y M} \phi} G_{M N}^{a} G^{a M N}\right],
\end{aligned}
$$

where $M$ and $N$ are 5-dimensional (5-D) spacetime indices, running from 0 to $4, g_{M N}$ is the spacetime metric, $V_{0}, \gamma, \lambda_{U}$ and $\lambda_{Y M}$ are real constants and $\kappa_{5}$ is the 5-D gravity constant. $\phi$ is a real scalar field, and $F_{M N}$ is the field strength of $\mathrm{U}(1)$ gauge field $A_{M}$, i.e. $F_{M N}=\partial_{M} A_{N}-\partial_{N} A_{M} . G_{M N}^{a}$ is the field strength of the Yang-Mills field $B_{M}^{a}\left(B \equiv B_{M} \mathrm{~d} x^{M}=\right.$ $\left.\tau^{a} B_{M}^{a} \mathrm{~d} x^{M}\right)$, where we have chosen the simplest Yang-Mills gauge group, $\mathrm{SU}(2)$, which satisfies $\left[\tau^{a}, \tau^{b}\right]=i \epsilon^{a b c} \tau^{c}$ and $\operatorname{Tr}\left(\tau^{a} \tau^{b}\right)=\delta^{a b} / 2$ where $\epsilon^{a b c}$ is fully antisymmetric tensor and $\epsilon^{123}=1$. Then

$$
G_{M N}^{a}=\partial_{M} B_{N}^{a}-\partial_{N} B_{M}^{a}-\epsilon^{a b c} B_{M}^{b} B_{N}^{c},
$$

where the gauge group adjoint indices, $a, b$ and $c$ run over 1 to 3. $g_{U}$ and $g_{Y M}$ are gauge couplings. We set the AdS radius $L=1$.

The bulk equations of motion are given by

$$
\begin{aligned}
X \equiv & \frac{1}{\sqrt{-g}} \partial_{M}\left(\sqrt{-g} g^{M N} \partial_{N} \phi\right)+V_{0} \gamma e^{\gamma \phi} \\
& -\frac{\kappa_{5}^{2} \lambda_{U}}{4 g_{U}^{2}} e^{\lambda_{U} \phi} F_{M N} F^{M N}
\end{aligned}
$$

$$
\begin{aligned}
& -\frac{\kappa_{5}^{2} \lambda_{Y M}}{4 g_{Y M}^{2}} e^{\lambda_{Y M} \phi} G_{M N}^{a} G^{a M N}=0 \\
Y^{N} \equiv & \frac{1}{\sqrt{-g}} \partial_{M}\left(\sqrt{-g} e^{\lambda_{U} \phi} F^{M N}\right)=0 \\
\mathcal{Y}^{a N} \equiv & \frac{1}{\sqrt{-g}} \partial_{M}\left(\sqrt{-g} e^{\lambda_{Y M} \phi} G^{a M N}\right) \\
& +e^{\lambda_{Y M} \phi} \epsilon^{a b c} G^{b M N} B_{M}^{c}=0 \\
W_{M N} \equiv & R_{M N}+\frac{V_{0}}{3} g_{M N} e^{\gamma \phi}-\frac{1}{2} \partial_{M} \phi \partial_{N} \phi \\
& -\frac{\kappa_{5}^{2}}{2 g_{U}^{2}} e^{\lambda_{U} \phi}\left(F_{P M} F_{N}^{P}-\frac{1}{6} g_{M N} F_{P Q} F^{P Q}\right) \\
& -\frac{\kappa_{5}^{2}}{2 g_{Y M}^{2}} e^{\lambda_{Y M} \phi}\left(G_{P M}^{a} G_{N}^{a P}-\frac{1}{6} g_{M N} G_{P Q}^{a} G^{a P Q}\right)=0 .
\end{aligned}
$$

We start with a solution having a generic hyperscaling violating factor $\alpha$ and a temporal anisotropy factor $z$. Such solutions already appeared in [13], an Einstein-dilaton theory with two different $U(1)$ gauge fields. This is a spatially isotropic solution. Our solution is obtained in the almost same way but we want to get spatial anisotropy on top of this. The ansatz is given by

$$
\begin{aligned}
\mathrm{d} s^{2}= & r^{2 \alpha}\left(-r^{2 z} f(r) \sigma^{2}(r) \mathrm{d} t^{2}+\frac{\mathrm{d} r^{2}}{r^{2} f(r)}\right. \\
& \left.+r^{2} h^{-4}(r) \mathrm{d} x_{1}^{2}+r^{2} h^{2}(r)\left(\mathrm{d} x_{2}^{2}+\mathrm{d} x_{3}^{2}\right)\right) \\
B^{a} \tau^{a}= & b(r) \tau^{3} \mathrm{~d} t+\omega(r) \tau^{1} \mathrm{~d} x_{1}, \\
A= & a(r) \mathrm{d} t \\
\phi= & \phi(r),
\end{aligned}
$$

where $\omega(r)$ represents the spatial anisotropy and $\tau^{a}=\frac{\sigma^{a}}{2}$. When there is no anisotropy, i.e. $\omega(r)=0$, the background geometry becomes that of 5-D charged black brane solutions, which are given by

$$
\begin{aligned}
f(r) & =1-m r^{-3 \alpha-z-3}+\frac{\kappa_{5}^{2}}{g_{Y M}^{2}} \tilde{\mu}^{2} r^{-6 \alpha-4-2 z}, \sigma(r)=1, \quad h(r)=1 \\
G_{r t}^{3} & =\partial_{r} b(r)=\tilde{\mu} e^{-\sqrt{\frac{\alpha+z-1}{6(\alpha+1)}} \phi_{0}} \sqrt{6(\alpha+1)(3 \alpha+z+1)} r^{-3 \alpha-z-2}, \\
G_{r x_{1}}^{1} & =0, \\
F_{r t} & =\partial_{r} a(r)=\frac{g_{U}^{2}}{\kappa_{5}^{2}} e^{\frac{2 \alpha+3}{\sqrt{6(\alpha+1)(\alpha+z-1)}} \phi_{0}} \sqrt{2(z-1)(3 \alpha+z+3)} r^{3 \alpha+z+2}, \\
\phi & =\phi_{0}+\sqrt{6(\alpha+1)(\alpha+z-1)} \ln r,
\end{aligned}
$$

where $m$ is the mass density of the black brane and $\tilde{\mu}$ is the chemical potential, and the free parameters of this model are fixed by

$$
V_{0}=(3 \alpha+z+2)(3 \alpha+z+3) e^{\gamma \phi_{0}}
$$


and

$$
\begin{aligned}
\gamma & =-\frac{\sqrt{2} \alpha}{\sqrt{3(\alpha+1)(\alpha+z-1)}}, \quad \lambda_{Y M}=\sqrt{\frac{2(\alpha+z-1)}{3(\alpha+1)}}, \\
\lambda_{U} & =-\frac{2(2 \alpha+3)}{\sqrt{6(\alpha+1)(\alpha+z-1)}} .
\end{aligned}
$$

We note that this solution should satisfy the null-energy condition [13],

$(\alpha+1)(\alpha+z-1) \geq 0$.

To be more precise, we consider a null vector in this black brane background as $\zeta^{M}=\left(\sqrt{g^{r r}}, \sqrt{g^{t t}}, 0,0,0\right)$; then

$T_{M N} \zeta^{M} \zeta^{N} \sim R_{r}^{r}-R_{t}^{t}=3(\alpha+1)(\alpha+z-1) r^{-2 \alpha} f(r) \geq 0$,

and this leads (16).

We would like to explore a spatial anisotropy in this background by turning on $B_{x_{1}}^{1}(=\omega(r))$ without considering its back reactions to the background geometry. ${ }^{5}$ This limit can be obtained by demanding that the Yang-Mill coupling is taken to be infinity, i.e. $\frac{\kappa_{5}^{2}}{g_{Y M}^{2}} \rightarrow 0$. In such a limit, spacetime becomes just AdS-Schwarzschild type black brane since the term that is proportional to the chemical potential in $f(r)$ disappears.

When we look at the action (6), it is clear that the YangMills fields can absorb the Yang-Mills coupling by a field redefinition as $\frac{1}{g_{Y M}} A_{M}^{a} \rightarrow A_{M}^{a}$. Therefore, the decoupling limit actually refers to the weak field limit of the Yang-Mills field whose stress-energy tensor is too small to back react to the other fields. In much literature, the classical (quantum) field theory in a robust background geometry is studied by ignoring back reaction from the fields and our analysis is along the same lines.

For further discussion, we rescale the radial coordinate $r$ by the size of the black brane horizon. More precisely, we define a new radial variable $u$ by

$r=r_{0} u$

where $r_{0}$ is for the horizon, which is obtained by

$f\left(r=r_{0}\right)=1-\frac{m}{r_{0}^{3 \alpha+z+3}}=0 ;$

then $m=r_{0}^{3 \alpha+z+3}$. Together with this, we rescale the other coordinate variables as $t \rightarrow r_{0}^{-z} t$ and $\left\{x_{1}, x_{2}, x_{3}\right\} \rightarrow$ $\frac{1}{r_{0}}\left\{x_{1}, x_{2}, x_{3}\right\}$. As a result, the background metric becomes

\footnotetext{
5 The genuine spatial anisotropy is encoded in the back reactions to the background spacetime, meaning that non-trivial $h(r)$ shows the anisotropy. It is certain that once we compute the back reactions to the metric, we must have non-trivial $h(r)$ and we will leave this project for future work.
}

$$
\begin{aligned}
\frac{\mathrm{d} s^{2}}{r_{0}^{2 \alpha}}= & u^{2 \alpha}\left(-u^{2 z} f(u) \mathrm{d} t^{2}+\frac{\mathrm{d} u^{2}}{u^{2} f(u)}\right. \\
& \left.+u^{2}\left(\mathrm{~d} x_{1}^{2}+\mathrm{d} x_{2}^{2}+\mathrm{d} x_{3}^{2}\right)\right)
\end{aligned}
$$

where

$f(u)=1-u^{-3 \alpha-z-3}$

Together with this, we define a new chemical potential, $\mu$, in this rescaled coordinate as

$\mu \equiv \frac{\tilde{\mu}}{r_{0}^{3 \alpha+z+1}} e^{-\sqrt{\frac{\alpha+z-1}{6(\alpha+1)}} \phi_{0}} \frac{\sqrt{6(\alpha+1)(3 \alpha+z+1)}}{3 \alpha+z+1}$.

Then the background value of $B_{t}^{3}$ becomes

$b(u)=\mu\left(1-u^{-3 \alpha-z-1}\right)$.

In this rescaled coordinate, the Yang-Mill equations are written as

$$
\begin{aligned}
\mathcal{Y}_{x}^{1}= & u^{-5 \alpha-z-2} \partial_{u}\left(u^{3 \alpha+3 z} f(u) \partial_{u} \omega(u)\right) \\
& +f^{-1}(u) u^{-2 \alpha-4} b^{2}(u) \omega(u)=0 \\
\text { and } \mathcal{Y}_{t}^{3}= & u^{-5 \alpha-z-2} \partial_{u}\left(u^{3 \alpha+z+2} \partial_{u} b(u)\right) \\
& -f^{-1}(u) u^{-2 \alpha-4} \omega^{2}(u) b(u)=0 .
\end{aligned}
$$

\section{Analytic and numerical approaches to the critical points}

In this section, we estimate the critical value of chemical potential for the generic $z$ and $\alpha$. It is more convenient to use the $\xi$ coordinate, where $\xi$ is given by $\xi=\frac{1}{u}$. In this coordinate, (24) and (25) are given by

$$
\begin{aligned}
0= & \frac{\mathrm{d}}{\mathrm{d} \xi}\left(\xi^{2-3 \alpha-3 z} f(\xi) \frac{\mathrm{d} \omega(\xi)}{\mathrm{d} \xi}\right) \\
& +f^{-1}(\xi) \xi^{-3 \alpha-z} b^{2}(\xi) \omega(\xi), \\
0= & \frac{\mathrm{d}}{\mathrm{d} \xi}\left(\xi^{-3 \alpha-z} \frac{\mathrm{d} b(\xi)}{\mathrm{d} \xi}\right) \\
& -f^{-1}(\xi) \xi^{-3 \alpha-z} \omega^{2}(\xi) b(\xi) .
\end{aligned}
$$

Near the critical point, we expect a second order phase transition. We assume that the amplitude of the field, $\omega(\xi)$, is small and it is suppressed by $\epsilon$, a dimensionless small constant. Then one can regard the solution of $b(\xi)$ to be approximately described by (23) in the new coordinate $\xi$ by

$b(\xi)=\mu\left(1-\xi^{3 \alpha+z+1}\right)+O\left(\epsilon^{2}\right)$.

Near the boundary of the spacetime, $\omega(\xi)$ will behave as

$\omega(\xi)=\epsilon\left\langle J_{x_{1}}^{1}\right\rangle \xi^{3 \alpha+3 z-1} F(\xi)+O\left(\epsilon^{3}\right)$, 
where $\left\langle J_{x_{1}}^{1}\right\rangle$ is an anisotropic order parameter and $F(\xi)$ is a new function that satisfies the boundary conditions

$F(0)=1$, and $F^{\prime}(0)=0$.

The factor $\xi^{3 \alpha+3 z-1}$ in (29) ensures its asymptotic behavior and we solve the equation up to $O(\epsilon)$

To estimate the critical values of chemical potential for various $\alpha$ and $z$, we use Sturm-Liouville technique. To solve the Sturm-Liouville problem, (26) should be written in the form

$0=\frac{\mathrm{d}}{\mathrm{d} \xi}\left(K(\xi) \frac{d F(\xi)}{\mathrm{d} \xi}\right)-P(\xi) F(\xi)+\mu^{2} Q(\xi) F(\xi)$,

where

$$
\begin{aligned}
K(\xi) & =\xi^{3 \alpha+3 z}\left(1-\xi^{3 \alpha+z+3}\right), \\
P(\xi) & =-(3 \alpha+3 z-1) \xi^{3 \alpha+3 z-1} \frac{\mathrm{d}}{\mathrm{d} \xi}\left(1-\xi^{3 \alpha+z+3}\right), \\
Q(\xi) & =\frac{\xi^{3 \alpha+5 z-2}\left(1-\xi^{3 \alpha+z+1}\right)^{2}}{1-\xi^{3 \alpha+z+3}} .
\end{aligned}
$$

One can estimate the chemical potential by obtaining its upper bounds by using the general variation method of the Sturm-Liouville problem. In the range of $0 \leq \xi \leq 1$, the eigenvalue $\mu^{2}$ in the equation is minimized by the following expression:

$\mu^{2}=\frac{\int_{0}^{1} \mathrm{~d} \xi\left[K(\xi)\left(\frac{d F(\xi)}{\mathrm{d} \xi}\right)^{2}+P(\xi) F^{2}(\xi)\right]}{\int_{0}^{1} \mathrm{~d} \xi Q(\xi) F^{2}(\xi)}$.

To estimate this effectively, we introduce a test function of $F(\xi)$ satisfying $(30)$ as

$F(\xi)=1-t \xi^{2}$

where $t$ is an arbitrary real constant to be determined under the condition that $\mu^{2}$ becomes minimum. The integrations can be analytically performed in the range of

$5 z+3 \alpha>1, \quad 3 \alpha+z>-3,4 z+6 \alpha>-2$ and $z+\alpha>-1$

and on top of this we also consider the null-energy condition (16). Only in this region, the Sturm-Liouville problem is well defined.

We study the minimum value of the chemical potential for various possible values of $z$ and $\alpha$. First of all, we evaluate the upper bounds of the critical value of the chemical potential for fixed $\alpha$. The results are shown in Fig. 1a with solid lines. There are five different graphs in it and from below, each solid line indicates the critical value of the chemical potential when $\alpha=0, \alpha=1 \ldots \alpha=4$ as $z$ continuously varies from 1 to 5 . The graphs show monotonically increasing behaviors as $z$ increases.

Especially, when $z=1$ and $\alpha=0$, we get $\mu_{\mathrm{c}}=4.09206$ by the Sturm-Liouville method. In [9], the authors address the subject of an Einstein-SU(2) Yang-Mills system in asymptotically AdS space showing a phase transition from the spatially isotropic phase to an anisotropic phase when $\mu_{\mathrm{c}}=4$. The critical value of the chemical potential is correct within $2.3 \%$ error.

Next, we study the chemical potential upper bounds for fixed $z$. The result is presented in Fig. 1b with solid lines too. For a large $z, \mu_{\mathrm{c}}$ seems to increase linearly as $z$ increases, but for the small $z$ region each graph may show a minimum of the critical value of chemical potential and it rebounds as $\alpha$ decreases.

\section{Numerics}

In this section, we also consider the limit that the YangMills coupling constant is large, $\frac{\kappa_{5}^{2}}{g_{Y M}^{2}} \rightarrow 0$, and there is no back reaction to the background geometry, the scalar field and the $U(1)$ gauge fields, namely we take the probe limit. We numerically find critical values of $\mu$ for the given values of $\alpha$ and $z$ and compare the results with the analytic ones obtained in Sect. 3. We calculate and compare free energy densities with/without turning on $\omega(\xi)$ by using the numerically obtained values of $\mu, \alpha$, and $z$.

\subsection{Numerical solutions of $\omega(\xi)$ and $b(\xi)$}

Boundary conditions In the asymptotic region (as $\xi \rightarrow 0$ ), the following boundary conditions are applied:

$\lim _{\xi \rightarrow 0} b(\xi) \rightarrow \mu, \quad \lim _{\xi \rightarrow 0} \omega(\xi) \rightarrow 0$,

and near the black brane horizon (as $\xi \rightarrow 1$ ), the boundary condition is

$b(\xi) \rightarrow 0$.

We employ the shooting method to find the values of $\mu$ and $z$ (or $\mu$ and $\alpha$ ) for a given value of $\alpha$ (or $z$ ) with these boundary conditions.

The numerical results and comparison with the SturmLiouville computations The numerical results are presented by the dashed line in Fig. 1. In Fig. 1, $\mu$ versus $\alpha$ and $\mu$ versus $z$ graphs are depicted in (a) and (b) for the given integral values of $z=1,2,3,4,5$ and $\alpha=0,1,2,3,4$, respectively. The solid lines present the results from the Sturm-Liouville 


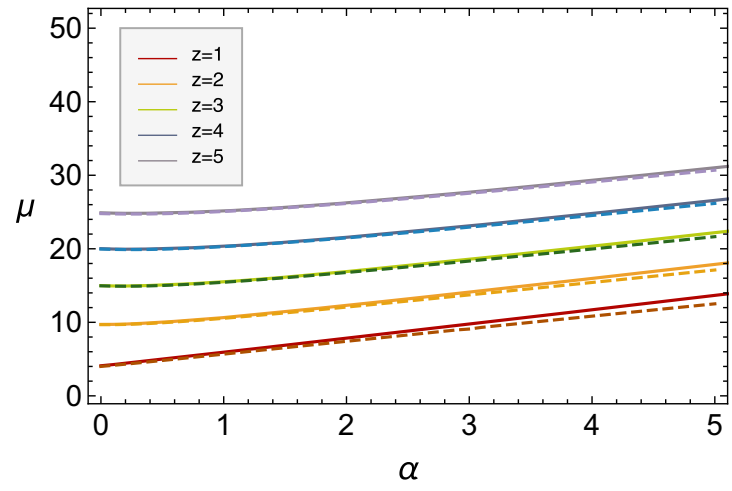

(a) For fixed $z$

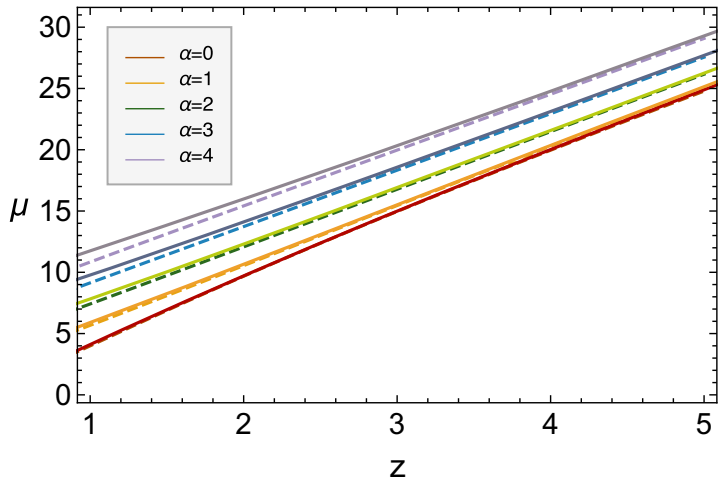

(b) For fixed $\alpha$

Fig. 1 Plot for the chemical potential $\mu$ versus $\alpha$ (or $z$ ) for the given values of $z$ (or $\alpha$ ). The solid lines show graphs of the upper bounds obtained from the analytic methods and the dashed lines present the numerical results

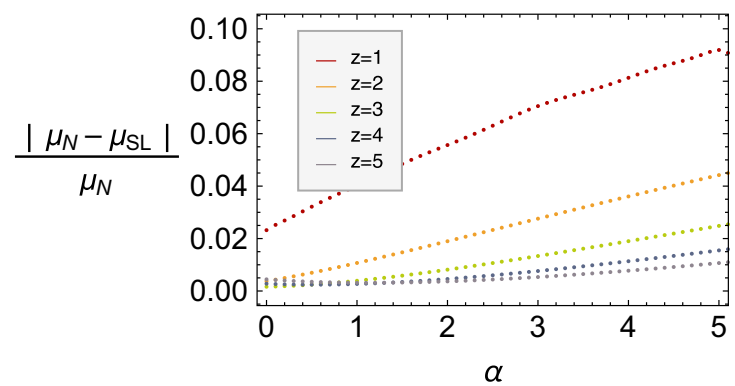

(a) For fixed $z$

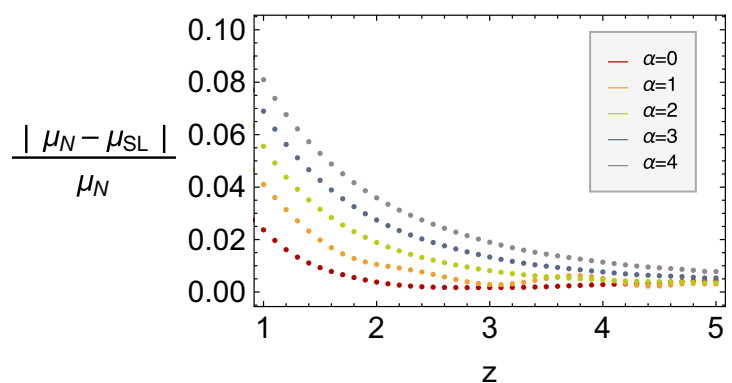

(b) For fixed $\alpha$

Fig. 2 A comparison of the values of $\mu$ in the Sturm-Liouville method and the numerical results

method and we show the dashed and solid lines together for comparison. For the given testing range of $\alpha$ and $z$, the discrepancy ratio ${ }^{6}$ between the numerical method and the Sturm-Liouville method for $\mu$ is less than 9.2 percents for $0<\alpha<4$ and $z=1,2,3,4$, and 5. It is less then 8.1 percents for $\alpha=1,2,3,4$ and 5 and $1<z<5$. Especially, for $z=1$ and $\alpha=0$ our numerical value of $\mu_{c}$ yields 3.999, which well agrees with the result in $[8,18]$. As seen in Fig. 2, the Sturm-Liouville results approach the numerical ones as $z$ becomes larger and $\alpha$ becomes smaller.

We do not understand why the errors between the chemical potentials, obtained by employing numerical and analytic methods, respectively, become larger as $\alpha$ increases or $z$ decreases. However, the errors may be due to the truncation of the polynomial in the function $F(\xi)$. Considering more appropriate test functions may enhance the accuracy of the Sturm-Liouville method. For example, one can go further by adding more terms like having higher powers of $\xi$ and find more accurate results.

The numerical solutions of the $\omega(\xi)$ and $b(\xi)$ fields The numerical solutions as regards $\omega(\xi)$ and $b(\xi)$ are demonstrated in Fig. 3.

\footnotetext{
${ }^{6}$ We define the discrepancy ratio $=\frac{\mu_{\text {numeric }}-\mu_{\text {Strum--Liuville }}}{\mu_{\text {numeric }}} \times 100 \%$.
}

\subsection{Free energy density}

Since we consider the probe limit, it is enough to inspect the Yang-Mills kinetic term in the background of other fields to examine the thermodynamic phase transition. The YangMills action with imaginary time obtained by a Wick rotation, $t=-i \tau$, is given by

$$
\begin{aligned}
S_{Y M}^{E}= & \frac{\beta V_{3}}{2 g_{Y M}^{2}} r_{0}^{3 \alpha+2 z-2} e^{\lambda_{Y M} \phi_{0}} \int_{0}^{1} \mathrm{~d} \xi \xi^{-3 \alpha-z} \\
& {\left[\xi^{2-2 z} f(\xi)\left(\frac{\mathrm{d} \omega(\xi)}{\mathrm{d} \xi}\right)^{2}-\frac{\omega^{2}(\xi) b^{2}(\xi)}{f(\xi)}-\left(\frac{\mathrm{d} b(\xi)}{\mathrm{d} \xi}\right)^{2}\right] } \\
& \equiv \frac{1}{g_{Y M}^{2}} r_{0}^{3 \alpha+2 z-2} e^{\lambda_{Y M} \phi_{0}} \mathcal{S}_{Y M}^{E}
\end{aligned}
$$

where $V_{3}$ is the coordinate volume of the spatial boundary. $\beta$ is the periodicity of the Euclidean time in the rescaled coordinate ( $\xi$ coordinate) and is identified with the inverse of the temperature. By using the equations of motion of the Yang-Mills fields, one can derive a simpler form of the Yang-Mills action. The free energy is defined by the Euclideanized on-shell action times the temperature, which is given by 


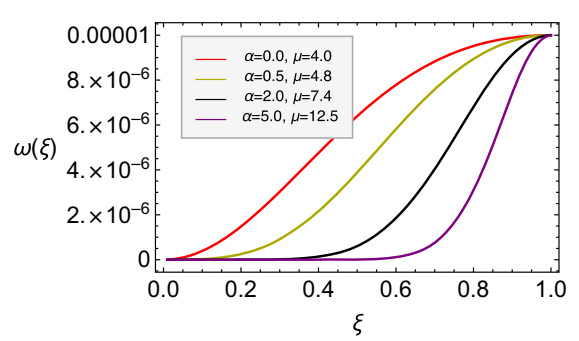

(a) For $z=1$

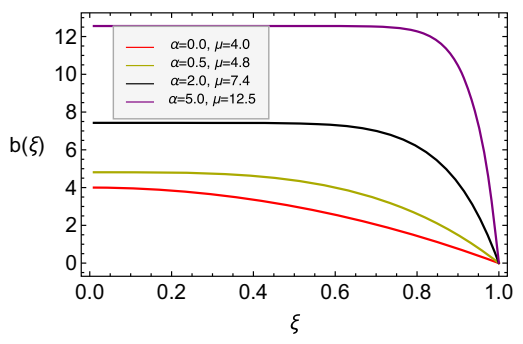

(d) For $z=1$

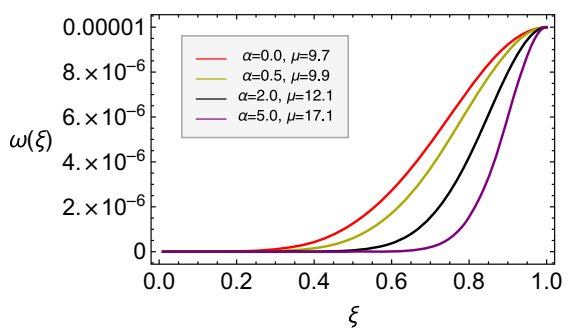

(b) For $z=2$

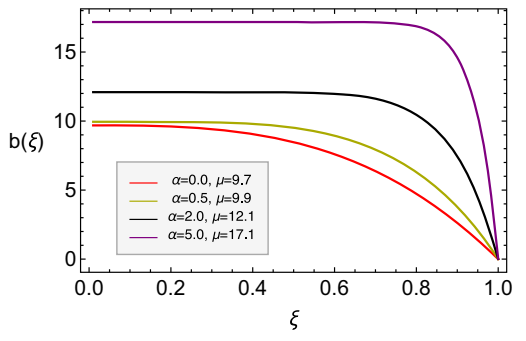

(e) For $z=2$

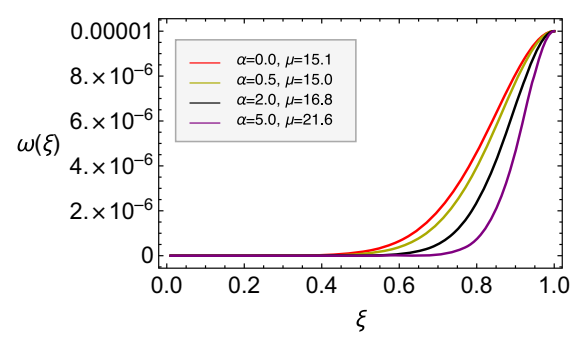

(c) For $z=3$

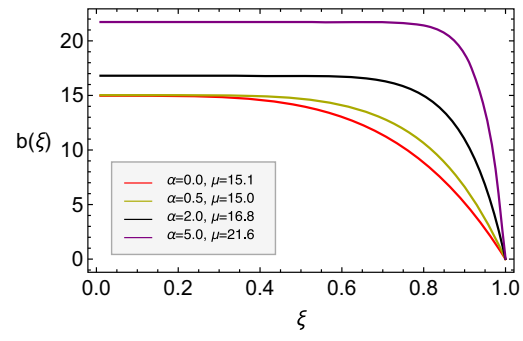

(f) For $z=3$

Fig. 3 Numerical fittings of $\omega(\xi)$ and $b(\xi)$ functions

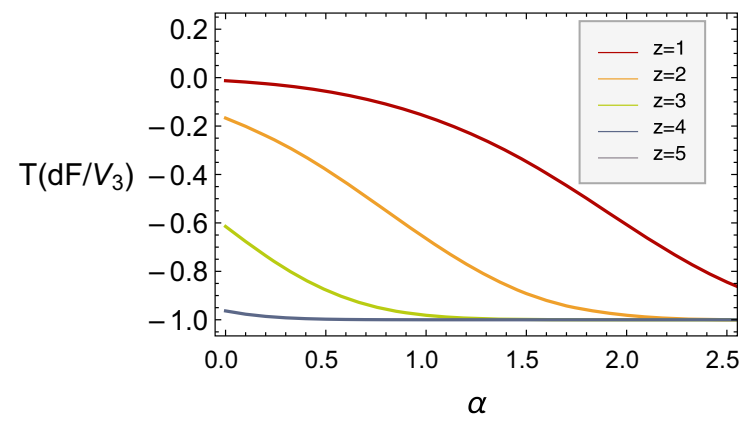

(a) For fixed $z$

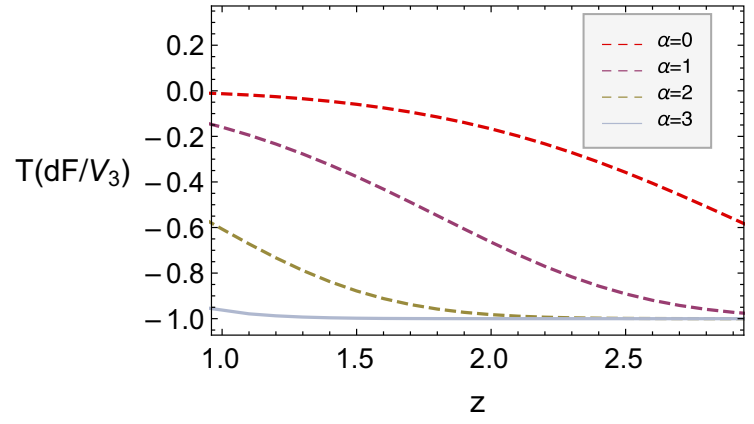

(b) For fixed $\alpha$

Fig. 4 The difference of free energy density between the anisotropic state $(\omega \neq 0)$ and the isotropic state $(\omega=0)$ for the fixed values of $z$ in a and $\alpha$ in $\mathbf{b}$

$$
\begin{aligned}
F_{Y M}= & T \mathcal{S}_{Y M}^{E}=\frac{V_{3}}{2}\left(\int_{0}^{1} \mathrm{~d} \xi \xi^{-3 \alpha-3 z+2} f(\xi)\left(\partial_{\xi} \omega(\xi)\right)^{2}\right. \\
& \left.+\left.\xi^{-3 \alpha-z} b(\xi) \partial_{\xi} b(\xi)\right|^{\xi=0}\right) .
\end{aligned}
$$

In the spatially isotropic phase, $\omega(\xi)=0, b(\xi)=\mu(1-$ $\xi^{3 \alpha+z+1}$ ) and so the free energy is given by

$$
\begin{aligned}
F_{Y M-\text { iso }} & =T \mathcal{S}_{Y M-\text { iso }}^{E} \\
& =-\mu^{2} \frac{V_{3}}{2} \int_{0}^{1} \mathrm{~d} \xi(3 \alpha+z+1)^{2} \xi^{3 \alpha+z}, \\
& =-\left.\mu^{2} \frac{V_{3}}{2}(3 \alpha+z+1)\left(1-\xi^{3 \alpha+z+1}\right)\right|^{\xi=0} .
\end{aligned}
$$

If we restrict our study to the region $3 \alpha+z+1>0$, then

$$
F_{Y M-\text { iso }}=-\mu^{2} \frac{V_{3}}{2}(3 \alpha+z+1) .
$$

If $\Delta F \equiv T \Delta S^{E}=T S_{Y M}^{E}-T S_{Y M \text {-iso }}^{E}<0$, the spatially anisotropic phase is more favored and then there will be a thermodynamic phase transition from the spatially isotropic phase to the anisotropic one.

To check the behavior of the free energy difference for the given testing ranges of $\alpha$ and $z$, we plot the free energy density difference by employing the following parameterization because the free energy itself shows a very large magnitude:

$$
\begin{aligned}
\mathrm{T}\left(\mathrm{d} F / V_{3}\right) \equiv & \tanh [\{\text { free energy density }(\omega \neq 0) \\
& - \text { free energy density }(\omega=0)\}] \\
= & \tanh \left[\left(F_{Y M}-F_{Y M-\text { iso }}\right) / V_{3}\right] .
\end{aligned}
$$

That $\left(\mathrm{d} F / V_{3}\right)$ is negative means that $F_{Y M}<F_{Y M-\text { iso }}$ and so the anisotropic phase is stable.

In Fig. 4a, a graph of $\mathrm{T}\left(\mathrm{df} / \mathrm{V}_{3}\right)$ versus $\alpha$ (in the range of $0 \leq \alpha \leq 2.5$ ) is given to show the free energy density differ- 


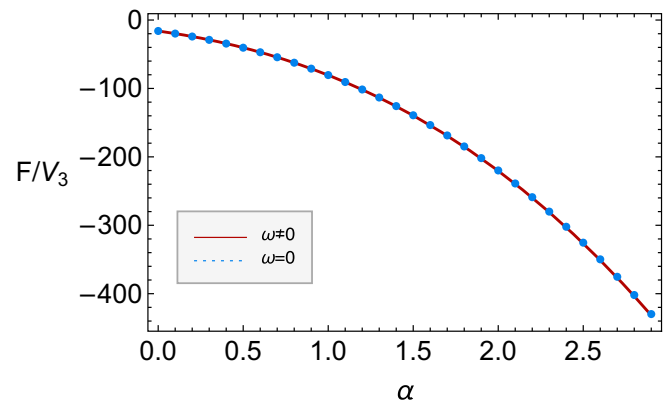

(a) When $z=1$, the free energy density depending on $\alpha$

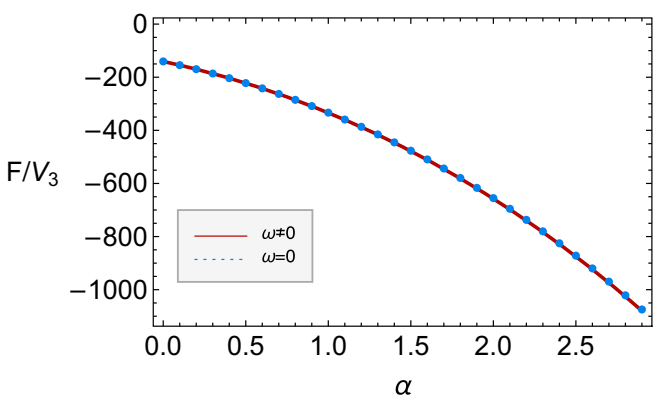

(c) When $z=2$, the free energy density depending on $\alpha$

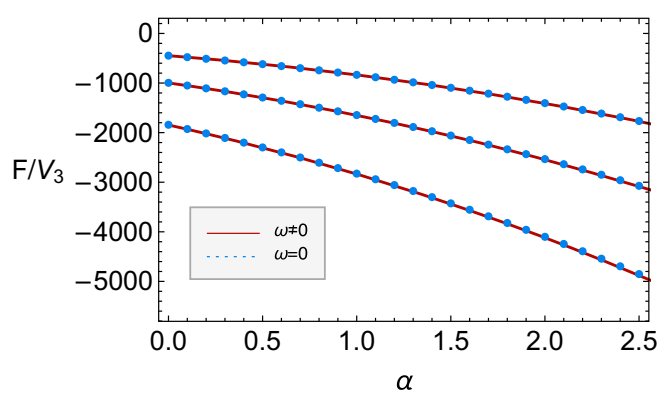

(e) When $z=3,4,5$ from top to bottom on the left axis. The free energy density depending on $\alpha$

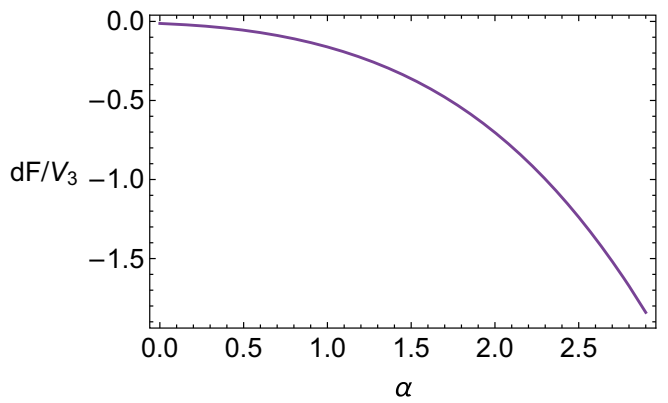

(b) When $z=1$, the difference of the free energy density depending on $\alpha$

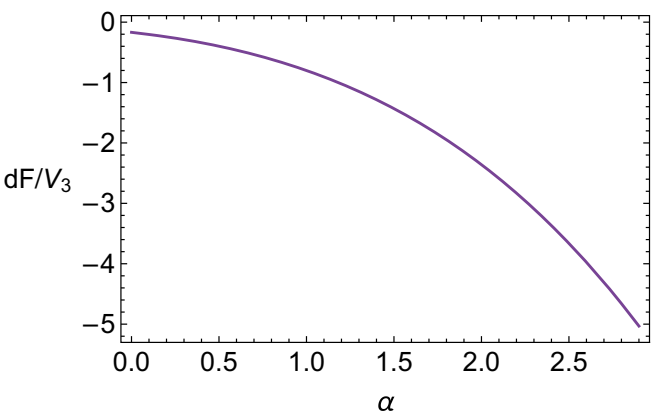

(d) When $z=2$, the difference of the free energy density depending on $\alpha$

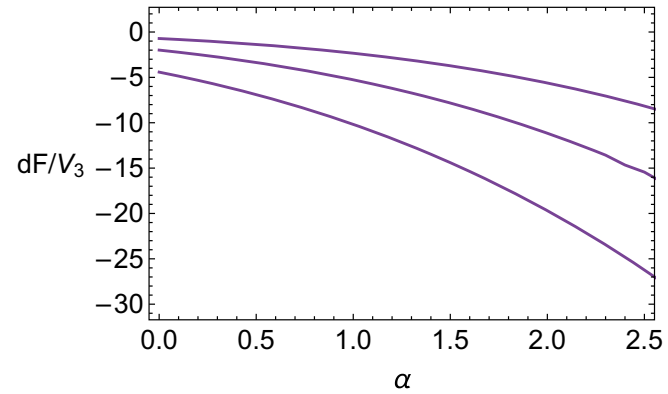

(f) When $z=3,4,5$ from top to bottom on the left axis, the difference of the free energy density depending on $\alpha$

Fig. 5 For fixed value of $z$. The free energy densities depending on $\alpha$ for the anisotropic and isotropic phase (left), and the free energy density difference between the two phases (right) depending on $\alpha$. Respectively, $\mathbf{a} \mathbf{b} z=1, \mathbf{c}, \mathbf{d} z=2, \mathbf{e}, \mathbf{f} z=3,4,5$ from top to bottom on the left axis

ence between the anisotropic and the isotropic phase for the given values of $z$ in our testing range $(1 \leq z \leq 3)$. T $\left(\mathrm{df} / \mathrm{V}_{3}\right)$ is negative from $\alpha=0$ to around $\alpha=2.5$ for the integral values of $z=1,2,3,4$ and 5 , but it is undetermined after $\alpha=2.5$ and so we do not plot it after $\alpha=2.5$. ${ }^{7}$ We conclude that, for the given values of $z=1,2,3,4$ and 5 and for the range of $0 \leq \alpha \leq 2.5$, the values of the chemical potential, $\mu_{\mathrm{c}}$, that we obtain in Fig. 1a are the critical values. Beyond this region, however, we do not have a definite conclusion.

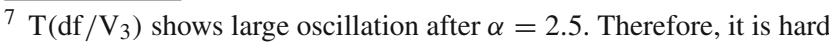
to distinguish the regions of anisotropic phase from the isotropic ones after this. We expect that this is due to numerical errors but we do not understand what makes such errors yet.
}

In Fig. $4 \mathrm{~b}$, a $\mathrm{T}\left(\mathrm{df} / \mathrm{V}_{3}\right)$ versus $z$ graph(in the range of $1 \leq z \leq 3)$ is displayed for the given integral values of $\alpha=0,1,2,3$ and 4 . For $\alpha=0,1,2, \mathrm{~T}\left(\mathrm{df} / \mathrm{V}_{3}\right)$ is negative when $1 \leq z \leq 5$. The chemical potential shown in Fig. $1 \mathrm{~b}$ is the critical value in $1 \leq z \leq 5$ for these values of $\alpha$. However, for $\alpha=3,4$, the free energy density difference, $\mathrm{d} F$, is negative up to a certain value of $\alpha$ and again shows large oscillating behaviors. Therefore, we conclude that for $\alpha=0,1,2,3$ and 4 , the values of the chemical potential, $\mu_{\mathrm{c}}$, that we obtain are the critical values.

A closer look at the free energy density for the given integral values of $z$ is in Fig. 5. The free energy densities for the anisotropic and isotropic phase are plotted together in 


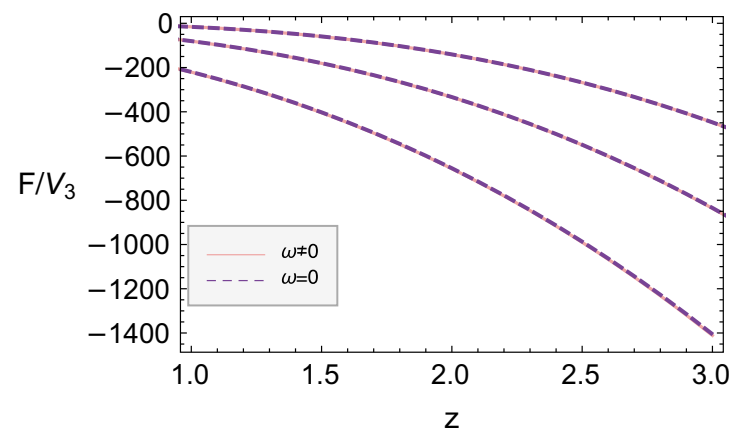

(a) When $\alpha=0,1,2$ (from top to bottom on the right axis), the free energy density depending on $z$

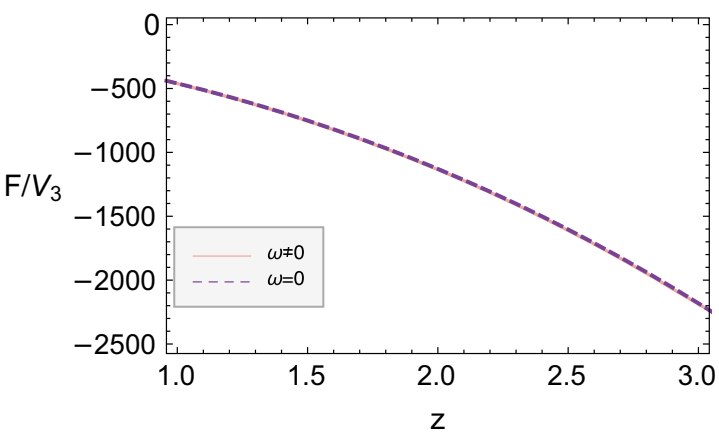

(c) When $\alpha=3$, the free energy density depending on $z$

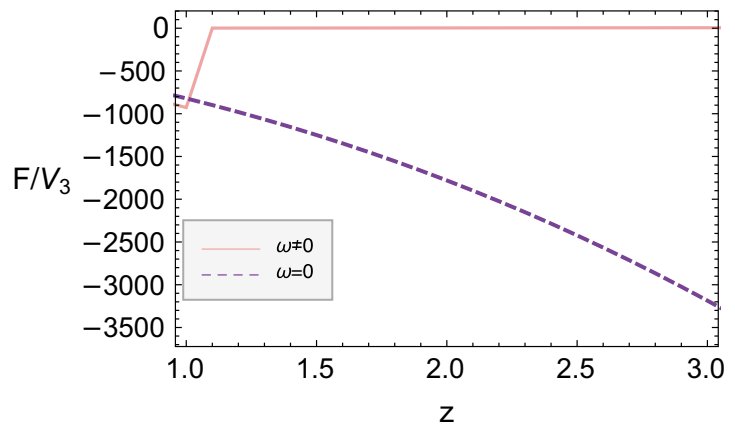

(e) When $\alpha=4$, the free energy density depending on $z$

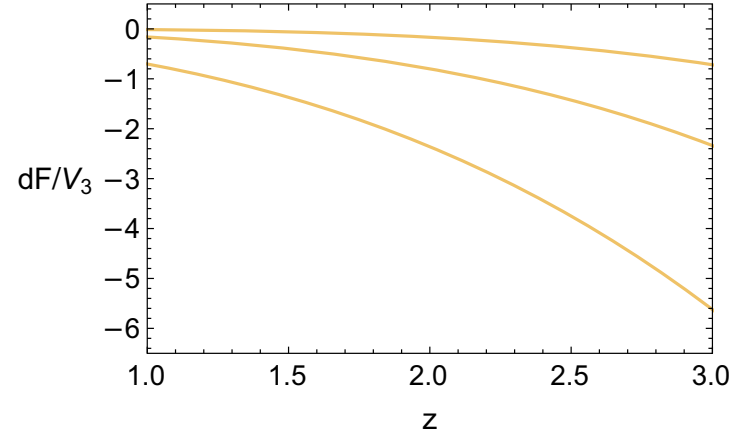

(b) When $\alpha=0,1,2$ (from top to bottom on the right axis), the difference of the free energy density depending on $z$

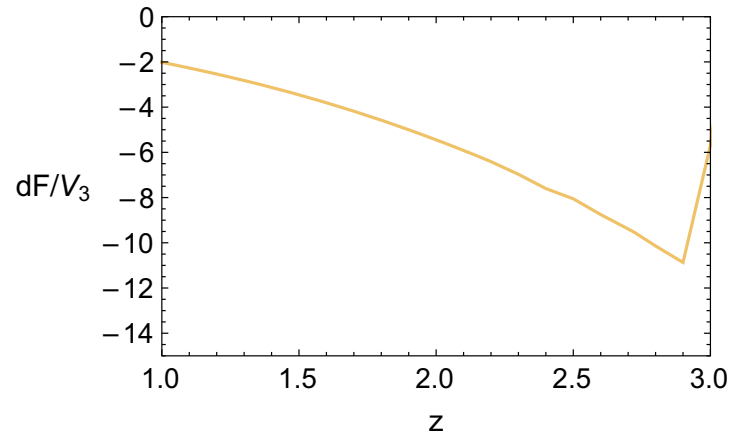

(d) When $\alpha=3$, the difference of the free energy density depending on $z$

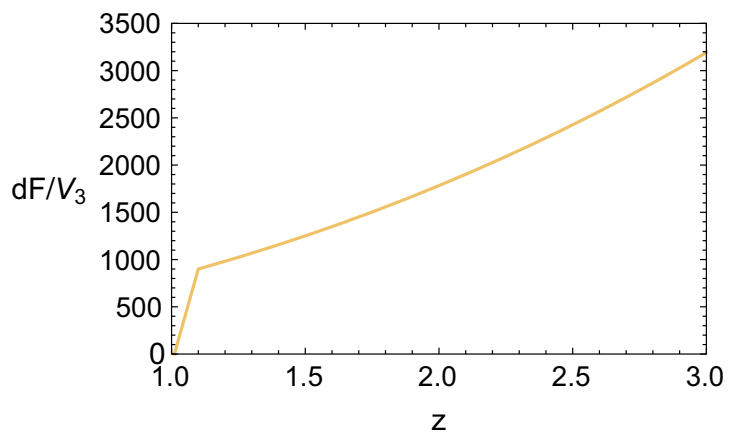

(f) When $\alpha=4$, the difference of the free energy density depending on $z$

Fig. 6 For fixed value of $\alpha$. The free energy densities for the anisotropic and isotropic phase (left), and the free energy density difference between two phases (right) depending on $z$. Respectively, $\mathbf{a}, \mathbf{b} \alpha=0,1,2$ (from top to bottom on the right axis), $\mathbf{c}, \mathbf{d} \alpha=3, \mathbf{e}, \mathbf{f} \alpha=4$

Fig. 5a, c, e. The red solid and the blue dashed lines indicate the anisotropic and isotropic phase, respectively. Their differences are shown in Fig. 5b, d, $\mathrm{f}$ in more detail.

For the fixed integral values of $\alpha$, the free energy densities are depicted in Fig. 6a, c, e, where the light red solid lines and the purple dashed lines are for the anisotropic and isotropic phase, respectively. When $\alpha=0,1,2, \mathrm{~T}\left(\mathrm{df} / \mathrm{V}_{3}\right)$ is negative in the entire region of our test range of $z$ and so the anisotropic phases are favored by the corresponding critical values of the chemical potential. However, for $\alpha=3$ in Fig. 6c, the free energy of the anisotropic phase is smaller than that of the isotropic phase when $1 \leq z \leq$ (roughly) 3 and so the anisotropic phase is favored in this region. After this, the isotropic phase is favored.

\section{Summary}

We explored thermodynamic phase transition between spatially isotropic and anisotropic phases of fluid dynamics by employing its gravity duals characterized by the hyperscaling violation factor $\alpha$ and the dynamical critical exponent $z$ 
when the current $\left\langle J_{1}^{x_{1}}\right\rangle$ starts to occur. We use analytic and numerical methods to find the critical value of $\mu$ for generic values of $\alpha$ and $z$, and check the thermodynamic stability of the anisotropic phase by calculating the free energy.

To do so, we employ its dual gravity action with the Einstein-dilaton-U(2) gauge fields and consider the probe limit of the Yang-Mills coupling constant being large, $\frac{\kappa_{5}^{2}}{g_{Y M}^{2}} \rightarrow 0$. We calculate the upper bounds of the critical value of the $\mu$ for the generic values of the $\alpha$ and $z$ by using the Sturm-Liouville method by using the test function (36). The result is displayed in Fig. 1 with the solid lines. We also calculate the critical value of $\mu$ by solving the coupled YangMills field equations numerically. With the choice of the magnitude of the vector order parameter of $\left\langle J_{1}^{x_{1}}\right\rangle \sim \epsilon=10^{-5}$, the shooting method searches the critical values of $\mu$ for the given values of $\alpha$ and $z$ satisfying appropriate boundary conditions. This result is shown in Fig. 1 with dashed lines. The two methods coincide within a few percentile errors, as illustrated in Fig. 2.

Next, we compute the free energy to check the thermodynamically favored phases among the spatially isotropic $(\omega=$ $0)$ and the anisotropic $(\omega \neq 0)$ phases. We investigate the region with $1 \leq z \leq 5$ and $0 \leq \alpha \leq 4$ and we found that the anisotropic phase is stable only for $0 \leq \alpha \leq$ (roughly) 3 for all values of $z$, and the isotropic phase is favored in the rest of the range of $\alpha$ and $z$. This result is shown in Figs. 4, 5, and 6.

For future work, it would be interesting to study the thermodynamic stability when considering the back reaction of Yang-Mills fields to the spacetime geometry, the dilaton and the gauge fields to study this system beyond the probe limit. Furthermore, the back reaction to the background metric will provide a computation of the shear viscosity and its holographic renormalization. As argued in [12], the shear viscosity of the anisotropic fluids runs as energy scale changes whereas almost all of the other holographic models for fluid dynamics give a trivial flow of the shear viscosity. Exploring this for the generic values of $z$ and $\alpha$ would be interesting.

Following the study in [19], the positivity of the canonical energy is equivalent to the dynamical instability and the canonical energy has a connection to the thermodynamic instability as well. Thus, checking the dynamical instability, such as the quasinormal modes of this gravitational system with or without back reaction, would also be interesting.

Acknowledgements M.P. is supported by a TJ Park Science Fellowship of the POSCO TJ Park Foundation. J.P. is supported by a Kwanjeong Fellowship of Kwanjeong Educational Foundation. J.H.O thanks W.J. This work was supported by a National Research Foundation of Korea (NRF) Grant funded by the Korea government (MSIP) (No. 2016R1C1B1010107).

Open Access This article is distributed under the terms of the Creative Commons Attribution 4.0 International License (http://creativecomm ons.org/licenses/by/4.0/), which permits unrestricted use, distribution, and reproduction in any medium, provided you give appropriate credit to the original author(s) and the source, provide a link to the Creative Commons license, and indicate if changes were made. Funded by SCOAP ${ }^{3}$.

\section{References}

1. J. Erdmenger, D. Fernandez, H. Zeller, JHEP 1304, 049 (2013). https://doi.org/10.1007/JHEP04(2013)049, arXiv:1212.4838 [hep-th]

2. S.A. Hartnoll, C.P. Herzog, G.T. Horowitz, JHEP 0812, 015 (2008) https://doi.org/10.1088/1126-6708/2008/12/015, arXiv:0810.1563 [hep-th]

3. S.S. Gubser, Phys. Rev. D 78, 065034 (2008). https://doi.org/10. 1103/PhysRevD.78.065034, arXiv:0801.2977 [hep-th]

4. S.S. Gubser, Phys. Rev. Lett. 101, 191601 (2008). https://doi.org/ 10.1103/PhysRevLett.101.191601, arXiv:0803.3483 [hep-th]

5. S. Kachru, X. Liu, M. Mulligan, Phys. Rev. D 78, 106005 (2008). https://doi.org/10.1103/PhysRevD.78.106005, arXiv:0808.1725 [hep-th]

6. P. Basu, J. He, A. Mukherjee, H.H. Shieh, JHEP 0911, 070 (2009). https://doi.org/10.1088/1126-6708/2009/11/070. arXiv:0810.3970 [hep-th]

7. O. Aharony, S.S. Gubser, J.M. Maldacena, H. Ooguri, Y. Oz, Phys. Rep. 323, 183 (2000). https://doi.org/10.1016/ S0370-1573(99)00083-6, arXiv:hep-th/9905111

8. G. Policastro, D.T. Son, A.O. Starinets, JHEP 0209, 043 (2002). https://doi.org/10.1088/1126-6708/2002/09/043, arXiv:hep-th/0205052

9. P. Basu, J.H. Oh, JHEP 1207, 106 (2012). https://doi.org/10.1007/ JHEP07(2012)106, arXiv:1109.4592 [hep-th]

10. P. Basu, J. He, A. Mukherjee, H.H. Shieh, Phys. Lett. B 689, 45 (2010). https://doi.org/10.1016/j.physletb.2010.04.042, arXiv:0911.4999 [hep-th]

11. M. Ammon, J. Erdmenger, V. Grass, P. Kerner, A. O'Bannon, Phys. Lett. B 686, 192 (2010). https://doi.org/10.1016/j.physletb.2010. 02.021, arXiv:0912.3515 [hep-th]

12. J.H. Oh, JHEP 1206, 103 (2012) https://doi.org/10.1007/ JHEP06(2012)103, arXiv:1201.5605 [hep-th]

13. M. Alishahiha, E.O. Colgain, H. Yavartanoo, JHEP 1211, 137 (2012). arXiv:1209.3946 [hep-th]

14. C. Charmousis, B. Gouteraux, B.S. Kim, E. Kiritsis, R. Meyer, JHEP 1011, 151 (2010). https://doi.org/10.1007/ JHEP11(2010)151, arXiv:1005.4690 [hep-th]

15. D.J. Griffiths, Introduction to quantum mechanics, 2nd edn. (Cambridge University Press, 2016) ISBN: 978-1-107-17986-8

16. H.-B. Zeng, X. Gao, Y. Jiang, H.-S. Zong, JHEP 1105, 002 (2011). arXiv:1012.5564 [hep-th]

17. D. Momeni, N. Majd, R. Myrzakulov, Europhys. Lett. 97, 61001 (2012). arXiv:1204.1246 [hep-th]

18. C.P. Herzog, S.S. Pufu, JHEP 0904, 126 (2009). arXiv:0902.0409 [hep-th]

19. S. Hollands, R.M. Wald, Commun. Math. Phys. 321, 629 (2013). https://doi.org/10.1007/s00220-012-1638-1, arXiv:1201.0463 [gr-qc]

20. S. Bhattacharyya, V.E. Hubeny, S. Minwalla, M. Rangamani, JHEP 0802, 045 (2008). https://doi.org/10.1088/1126-6708/2008/ 02/045, arXiv:0712.2456 [hep-th]

21. T. Fischer, L.C. Vink, J. Phys. Condens. Matter 23, 234117 (2011). https://doi.org/10.1088/0953-8984/23/23/234117, arXiv: 1010.3583 
22. A. Lucas, S. Sachdev, K. Schalm, Phys. Rev. D 89(6), 066018 (2014). https://doi.org/10.1103/PhysRevD.89.066018, arXiv:1401.7993 [hep-th]

23. P. Fonda, L. Franti, V. Kernen, E. Keski-Vakkuri, L. Thorlacius, E. Tonni, JHEP 1408, 051 (2014) https://doi.org/10.1007/ JHEP08(2014)051, arXiv:1401.6088 [hep-th]

24. J. Gath, J. Hartong, R. Monteiro, N.A. Obers, JHEP 1304, 159 (2013). https://doi.org/10.1007/JHEP04(2013)159, arXiv: 1212.3263 [hep-th]

25. B. Gouteraux, E. Kiritsis, JHEP 1304, 053 (2013). https://doi.org/ 10.1007/JHEP04(2013)053, arXiv:1212.2625 [hep-th]
26. N. Iizuka, S. Kachru, N. Kundu, P. Narayan, N. Sircar, S.P. Trivedi, H. Wang, JHEP 1303, 126 (2013). https://doi.org/10.1007/ JHEP03(2013)126, arXiv:1212.1948 [hep-th]

27. J. Bhattacharya, S. Cremonini, A. Sinkovics, JHEP 1302, 147 (2013). https://doi.org/10.1007/JHEP02(2013)147, arXiv:1208.1752 [hep-th]

28. E. Perlmutter, JHEP 1206, 165 (2012). https://doi.org/10.1007/ JHEP06(2012)165, arXiv:1205.0242 [hep-th] 